\title{
ON A REMARKABLE CLASS OF ENTIRE FUNCTIONS*
}

BY

\section{J. I. HUTCHINSON}

The problem treated in the following pages was first studied by Laguerret in this form. Let

$$
P_{n}(x)=A_{0 n}+A_{1 n} x+A_{2 n} x^{2}+\cdots+A_{n n} x^{n} \quad(n=1,2, \ldots)
$$

represent a sequence of polynomials which converges towards an entire function $F(x)$ as a limit. Suppose, further, that, for each value of $n, P_{n}(x)$ has all its roots real. Laguerre proved that $F(x)$ can be expressed as a canonical product, of genus not greater than 1 , multiplied by an exponential of the form $e^{a x^{s}+b x+c}$. If the additional assumption be made that all the roots of all the polynomials have the same sign (either all positive, or all negative) then the canonical product is of genus 0 and $a=0$.

In 1907 Petrovitch $\ddagger$ took up the problem in a more restricted form by assuming that the polynomials $P_{n}(x)$ are the sections of the power series for the limiting function. That is, if we write

$$
F(x)=a_{0}+a_{1} x+\cdots+a_{n} x^{n}+\cdots .
$$

then

$$
P_{n}(x)=F_{n}(x)=a_{0}+a_{1} x+\cdots+a_{n} x^{n}
$$

The coefficients then satisfy the necessary inequalities

$$
(n-1) a_{n-1}^{2}-2 n a_{n} a_{n-2} \geqq 0 \text {. }
$$

The conditions that $F_{n}(x)$ and $F(x)$ shall have all their roots real are given by Petrovitch in this way. Let $\Delta\left(a_{0}, a_{1}, \ldots, a_{n-1}, a_{n}\right)$ be the discriminant of $F_{n}(x)$ with $a_{0}, a_{1}, \ldots, a_{n-1}$ given and $a_{n}$ to be determined. Then $a_{n}$

* Presented to the Society, April 28, 1923.

† Sur les fonctions de genre un ou de genre zéro, Oeuvres I, p. 174.

¥Une classe remarquable de séries entières, Atti del IV Congresso internationale dei Matematici, Rome, 1908, pp. 36-43. 
can have any value between the least negative and the least positive root of the equation $\Delta=0$ with $a_{n}$ regarded as the unknown. We have here an infinity of conditions for the step by step determination of the limits between which $a_{2}, a_{3}, \ldots$, can be arbitrarily chosen, $a_{0}$ and $a_{1}$ being unrestricted. These conditions being in a form impossible to use for the effective construction of such series, Petrovitch refers to a paper by Mr. E. G. Hardy* for definite examples of series having the required property and then shows how, according to Laguerre, we may operate on any of these to obtain additional series in unlimited number.

After these general results, the remainder of Petrovitch's paper is devoted to a detailed study of the case $a_{n} \geqq 0, n=0,1, \ldots$, so that all the roots of $F_{n}(x)$ and of $F(x)$ are of the same (negative) sign. Quoting Laguerre he erroneously concludes that $F(x)$ is of the form mentioned above without observing that in his restricted case $b$ (as well as $a$ ) is necessarily zero. In fact, as Petrovitch shows, the conditions (2) are equivalent to

$$
\ddot{\sqrt{a_{n}}}<\frac{e a_{1}}{(n+1)(\sqrt{2})^{n}}
$$

From these conditions we may at once conclude from a well known theoremt that the genus of all, functions of the Petrovitch class u:hose roots are all of the same sign is zero.

The chief object of the present paper is to extend the results obtained by Hardy. We assume, with Hardy, that the coefficients $a_{n}$ in (1) are of the form

$$
a_{n}=\frac{1}{b_{1} b_{2} \ldots b_{n}}
$$

the $b_{n}$ being all positive and $a_{0}=1$ for convenience. It should be observed that the coefficients of any series may be represented in the form (3). If $\lim b_{n}=L$, then the radius of convergence is $L$. In order to exclude all $n \rightarrow \infty$

but entire functions from consideration, we assume $L=\infty$.

It is remarkable that, while the conditions on the $a_{n}$, as given by Petrovitch, are impossible of solution, the form given to the coefficients by Hardy enables

* On the zeros of a class of integral functions, Messenger of Mathematics, vol. 34 (1904), pp. 97-101.

$\dagger$ See, for example, E. Lindelöf, Mémoire sur la théorie des fonctions entières, Acta Societatis Scientiarum Fennicae, vol. 31, Art. 20, p. 46. 
the required conditions to be put in an extraordinarily simple form, requiring, however, a slight restriction upon the complete generality of Petrovitch's problem.

Hardy assumes $b_{n} \geqq 9 b_{n-1}$ and proves that the function $f(x)=1+t_{1}+t_{2}$ $+\ldots+t_{n}+\ldots, t_{n}=a_{n} x^{n}$, can be put in the form $t_{n}\left(1+\Phi_{n}\right),\left|\Phi_{n}\right|<1$, on certain circles $|x|=r_{n}, n=1,2, \ldots$, and hence $f(x)$ has exactly $n$ zeros inside of the circle $|x|=r_{n}$. These zeros are then proved to be real and negative.

We will now assume the more general condition

$$
b_{n} \geqq \alpha b_{n-1}, \quad \alpha \text { real and positive. }
$$

Hardy's method is applicable if $\alpha \geqq 4.8106,{ }^{*}$ but is useless for smaller values of $\alpha$. We accordingly adopt a different method which will apply to all cases to which condition (4) is applicable and which will possess the additional advantage of being extremely simple and elementary in character. It will be found that the least value $\alpha$ can have is 4 , since in that case we are able to prove the following theorem.

A. The relations

$$
b_{n} \geqq 4 b_{n-1} \quad(n=2,3, \ldots),
$$

are the necessary and sufficient conditions that the series $f(x)$ may have the properties:

1. The roots of $f(x)$ are all real, simple, and negative.

2. The roots of any polynomial $a_{m} x^{m}+\cdots+a_{n} x^{n}$ formed by taking any number of consecutive terms of $f(x)$ are all real, simple, and negative (excepting $x=0$ ).

3. As a special case of number 2 , any series $f_{n}(x)$ formed by taking the first $n+1$ terms of $f(x)$ has all its roots real, simple, and negative.

Introduce, for convenience, the notation

whence

$$
\pi_{n}=b_{1} b_{2} \cdots b_{n}
$$

$$
f(x)=1+\frac{x}{\pi_{1}}+\frac{x^{2}}{\pi_{2}}+\cdots+\frac{x^{n}}{\pi_{n}}+\cdots=1+t_{1}+\cdots+t_{n}+\cdots
$$

We observe that formula (5) is the necessary and sufficient condition that the polynomial $t_{n-2}+t_{n-1}+t_{n}$, formed by any three consecutive terms of $f(x)$, shall have real roots. Hence (5) is a necessary condition for Theorem $A$.

* This is (approximately) the lower limit of $a$ for which $\left|\Phi_{n}\right|<1$. 
To prove (5) a sufficient condition in all cases, we proceed to locate the roots of $f(x)$ and of $f_{n}(x)$. Give to $x$ the values

$$
x=-\sqrt{b_{2 \nu-1} b_{2 \nu}} \quad(\nu=1,2, \ldots)
$$

On account of the relations

$$
t_{n}=t_{n-1} \cdot \frac{x}{b_{n}}, \quad \frac{x}{b_{n}}=-\sqrt{\frac{b_{2 \nu-1}}{b_{n}} \cdot \frac{b_{2 \nu}}{b_{n}}}
$$

the terms in $f(x)$ will be alternately positive and negative and increasing numerically, as long as $n \leqq 2 v-1$, until we reach the numerically largest term, which is $\frac{x^{2 \nu-1}}{\pi_{2 \nu-1}}$. Consider the sum of the largest term and the two adjacent terms, viz.,

$$
\frac{x^{2 \nu-2}}{\pi_{2 \nu-2}}+\frac{x^{2 \nu-1}}{\pi_{2 \nu-1}}+\frac{x^{2 \nu}}{\pi_{2 \nu}}=T
$$

The first and last terms are equal, for the given value of $x$, and hence

$$
T=\frac{x^{2 \nu-2}}{\pi_{2 \nu-2}}\left(2-\sqrt{\frac{b_{2 \nu}}{b_{2 \nu-1}}}\right) \leqq 0,
$$

from (5). Accordingly, when $x$ has any one of the values (6), $f(\dot{x})$ may be written

$f(x)=\left(1+t_{1}\right)+\left(t_{8}+t_{3}\right)+\cdots+\left(t_{2 \nu-4}+t_{2 \nu-8}\right)+T+\left(t_{2 v+1}+\cdots\right)<0$.

The inequality follows from the fact that each group in parenthesis is negative. The last group, in particular, being the remainder of the series and consisting of terms alternately negative and positive and steadily decreasing numerically, is also negative.

In a similar manner, when $x$ has the values

$$
x=-\sqrt{b_{2 \nu} b_{2 \nu+1}} \quad(\nu=1,2 \ldots),
$$


$f(x)$ may be arranged in the form

$$
\begin{aligned}
f(x)=1+\left(t_{1}+t_{8}\right)+\cdots+ & \left(t_{2 \nu-8}+t_{2 \nu-2}\right) \\
& +t_{2 \nu-1}\left(2-\sqrt{\frac{b_{2 \nu+1}}{b_{2 \nu}}}\right)+\left(t_{2 \nu+2}+\cdots\right)>0,
\end{aligned}
$$

each group of which is positive, and hence the inequality. We have thus proved the theorem:

$B$. The series $f(x)$ has an infinity of real, negative zeros. An odd number of these zeros are situated between any two consecutive numbers of the series

$$
0, \quad-\sqrt{b_{1} b_{2}}, \quad-\sqrt{b_{2} b_{3}}, \ldots-\sqrt{b_{n-1} b_{n}}, \quad-\sqrt{b_{n} b_{n+1}}, \ldots
$$

It remains to prove that the zeros thus determined are all simple, that $f(x)$ has no other zeros, real or imaginary, and that just one root of $f(x)$ occurs betiveen any two consecutive numbers of the series (8). This is done by showing that the polynomials $f_{n}(x)$ have only real and simple zeros which are separated by the first $n+1$ numbers of the series (8). We proceed exactly as with $f(x)$ to substitute for $x$ the series of numbers (6), or (7), and to group the terms into negative, or positive groups, the only difference being that, when $2 \nu>n$, $f_{n}(x)$ will preserve an invariable sign which will be that of $(-1)^{n}$. Hence the result:

C. $f_{n}(x)$ has $n$ real, distinct, and negative roots which are separated by the numbers

$$
0, \quad-\sqrt{b_{1} b_{2}}, \quad-\sqrt{b_{2} b_{3}}, \ldots,-\sqrt{b_{n} b_{n+1}} .
$$

We prove at the same time, by this method, that the polynomial

$$
a_{n} x^{n}+a_{n+1} x^{n+1}+\cdots+a_{p} x^{p}
$$

formed by any number of consecutive terms of $f(x)$ has all its roots real, distinct, and negative (except for a multiple root $x=0$ ), since, if we remove the factor $a_{n} x^{n}$, the coefficients of the resulting polynomial will again be of the form (3), and will satisfy the required conditions (5).

Since $f(x)$ is the limit of $f_{n}(x)$, the conclusion, stated above, is proved. 
Example 1. By specializing condition (4), restricted cases of particular interest may be obtained. The most obvious procedure would be to drop the inequality sign and replace $\alpha$ by a chosen function of $n$ of such a nature as to satisfy (5) for all values of $n$. As a first example, let $\alpha$ be constant so that we have $b_{n}=\alpha b_{n-1}, n=1,2, \ldots, b_{0}=1$, whence $b_{n}=\alpha^{n}$. The resulting series is

$$
\varphi(x)=1+\frac{x}{\alpha}+\frac{x^{2}}{\alpha^{3}}+\cdots+\frac{x^{n}}{\alpha^{n(n+1) / 2}}+\cdots
$$

Substitute $x=-\alpha^{\nu}, \nu=1,2, \ldots$ The $(n+1)$ th term becomes

$$
(-1)^{n} \alpha^{n \nu-n(n+1) / 2}
$$

Assume $n \nu-\frac{n(n+1)}{2}=0$. whence $n=2 \nu-1$, and accordingly the $(n+1)$ th term reduces to $(-1)$. It is easy to verify that all the first $(n+1)$ terms of $\varphi(x)$ cancel each other, the $p$ th power of $x$ cancelling the $(2 \nu-1-p)$ th power, and hence $\varphi(x)$ reduces to

$$
\varphi\left(-\alpha^{\nu}\right)=\frac{1}{\alpha^{\nu}}-\frac{1}{\alpha^{2 \nu+1}}+\cdots
$$

Since $\alpha \geqq 4$, it follows that $\varphi\left(-\alpha^{\nu}\right)$ has a very small positive value less than $\alpha^{-\nu}$ which approaches zero as $\nu$ increases indefinitely. In other words, the roots of $\varphi(x)$ are represented approximately and asymptotically by the series of numbers $-\alpha,-\alpha^{2}, \ldots,-\alpha^{\nu}, \ldots$ This result is evidently true if $\alpha$ has any real or imaginary value such that $|\alpha|>1$.

The function $\varphi(x)$ satisfies the functional equation

$$
\varphi(\alpha x)-x \varphi(x)=1 .^{*}
$$

Denoting the function (9) by $\varphi_{1}(x)$, we observe that the function

$$
\varphi_{2}(x)=-\frac{1}{x}-\frac{1}{\alpha x^{2}}-\frac{1}{\alpha^{3} x^{3}}-\cdots-\frac{1}{\alpha^{n(n-1) / 2} x^{n}}-\cdots
$$

* This relation was brought to my attention by Professor W. A. Hurwitz. 
also satisfies (10). The most general solution of (10) would then be given by the formula

$$
\varphi(x)=c \varphi_{1}(x)+(1-c) \varphi_{2}(x),
$$

in which $c$ is a constant, or a function of $x$ satisfying the functional equation $c(\alpha x)=c(x)$.

Example 2. Assume $b_{n}=a^{n}-1, n=1,2, \ldots$, that is, assume

$$
b_{n}=\alpha_{n} b_{n-1}, \quad \alpha_{n}=\frac{a^{n}-1}{a^{n-1}-1} .
$$

We then obtain

$$
\begin{aligned}
& f(x)=1+\frac{x}{a-1}+\frac{x^{2}}{(a-1)\left(a^{2}-1\right)} \\
& \quad+\cdots+\frac{x^{n}}{(a-1)\left(a^{8}-1\right) \cdots\left(a^{n}-1\right)}+\cdots \\
& \quad=\left(1+\frac{x}{a}\right)\left(1+\frac{x}{a^{2}}\right) \cdots\left(1+\frac{x}{a^{n}}\right) \cdots,
\end{aligned}
$$

both expressions being convergent for all values of $x$ if $|a|>1$. If $|a|<1$, the series will be convergent for $|x|<1$. The zeros of this function are evident. When $a$ is real and positive, $f(x)$ will belong to the class designated in Theorem $A$, if $a \geqq 4$, since $\frac{a^{n}-1}{a^{n-1}-1}>a$.

If property no. 2 be omitted from Theorem $A$, the $b$ 's may have values somewhat smaller than those determined by (5), as Petrovitch shows by direct calculation. If we assume $b_{n}=\alpha_{n} b_{n-1}, \alpha_{n}$ being an unknown function of $n$ whose values for $n=2,3, \ldots$ are the smallest possible for which Theorem $A 1$ and 3 will be satisfied, then the results as far as known are: $\alpha_{2}=4, \alpha_{3}=3.375, \alpha_{4}=3.264$. It would seem that $\alpha_{n}$ is decreasing steadily to some unknown limit. All that we can say about such a limit is that it must be $\geqq 2$. For the coefficients in (1), as Petrovitch has pointed out, must satisfy (2), which is the necessary and sufficient condition that the $(n-2)$ nd derivative of $f_{n}(x)$ shall have real roots. Expressing the $a$ 's in terms of the $b$ 's by means of (3), formula (2) reduces to the simpler expression

$$
b_{n} \geqq \frac{2 n}{n-1} b_{n-1} .
$$


Comparing with the values given above for $\alpha_{n}$, we see that the equality sign is allowable in (11) for $n=2$, but for $n=3$ or 4 the inequality sign must be used. So that for all cases,

$$
\alpha_{n} \geqq \frac{2 n}{n-1}, \text { and hence } \lim _{n \rightarrow \infty} \alpha_{n} \geqq 2 \text {. }
$$

Denoting the moduli of the roots of $f(x)$ by $r_{1}, r_{2}, \ldots, r_{n}, \ldots$, we have from (7)

$$
r_{n}>\sqrt{b_{n-1} b_{n}} \geqq b_{1} a^{n-\frac{3}{2}}
$$

whence

$$
\sum_{n=1}^{\infty} \frac{1}{r_{n}^{\rho}}<\left(\frac{\alpha^{\frac{3}{2}}}{b_{1}}\right)^{\rho} \sum_{n=1}^{\infty} \frac{1}{\alpha^{\rho n}}, \quad \alpha \geqq 4
$$

Since the right member is convergent, however small the positive number $\rho$ may be, it follows that the order $\varrho$ of $f(x)$ is zero.

Formula (5), expressed in terms of the coefficient $a_{n}$, becomes

$$
a_{n-1}^{2}-4 a_{n} a_{n-2} \geq 0 \text {. }
$$

From this we readily deduce

$$
a_{n} \leqq \frac{a_{0}}{2^{n(n-1)}}\left(\frac{a_{1}}{a_{0}}\right)^{n},
$$

a formula that gives fairly precise information about the rate of growth of the maximum modulus of functions of the type included in Theorem $A$. This formula may advantageously replace the less simple formula of Petrovitch for all functions of the Petrovitch class having only positive coefficients, excepting the small residual class mentioned above in which not all of the $b_{n}$ satisfy (5).

CORNELL UNIVERSITY.

ITHACA, N.Y. 